\title{
LE RÉGIME MÉDITERRANÉEN DANS LA PRESSE QUOTIDIENNE NATIONALE FRANÇAISE
}

\begin{abstract}
Brigitte JUANALS $^{1}$
Le régime méditerranéen fait référence à un ensemble de traditions culinaires et culturelles issues des pays du bassin méditerranéen. L'analyse du traitement médiatique du régime méditerranéen est centrée sur la distribution et la circulation des discours, des représentations sociales et des acteurs mis en visibilité dans la presse. La méthodologie associe une approche quantitative et statistique à une approche qualitative ancrées toutes deux dans l'étude des discours. Sur une période de vingt ans (de 2000 à 2019), il apparaît que le régime méditerranéen constitue un thème d'intérêt suivi dans la durée par la presse nationale française selon cinq orientations thématiques dominantes et un univers lexical de nature patrimoniale, culinaire et environnementale, scientifique et sanitaire. Un nouvel univers lexical et de sens autour des végétaux émerge, porteur de représentations et de valeurs positives à l'intersection de la gastronomie, de la production locale et de préoccupations écologiques.
\end{abstract}

\section{Introduction}

Cet article porte sur le régime méditerranéen, également nommé " diète méditerranéenne ", une expression dans laquelle le terme

1 Brigitte Juanals est professeure des Universités en Sciences de l'information et de la communication à l'Université d'Aix-Marseille, École de Journalisme et de Communication (EJCAM), chercheuse au Centre Norbert Elias (UMR 8562).

Recherches en communication, $n^{\circ} 48$ - Article publié le 14/07/2020 
« diète », dérivé du grec diaita, signifie « un mode de vie, une communication entre l'esprit, le corps et le milieu » (González Turmo, 2012, p. 122). Le régime méditerranéen fait référence à un ensemble de traditions professionnelles, culinaires et culturelles ancrées dans les pays du bassin méditerranéen (Poulain, 2002). Il associe deux enjeux principaux, d'une part la « patrimonialisation des traditions culinaires 》 et, d'autre part, la « médicalisation de l'alimentation » (Bevilacqua, 2015, p. 316) qui apparaissent dans les discours tenus à son propos dans l'espace public (Marques da Silva, 2015). L'Unesco' a inscrit la « diète méditerranéenne » au patrimoine culturel immatériel de l'humanité le 16 novembre 2010. Cette décision est le résultat d'une « intense activité diplomatique et de divulgation scientifique » (Bevilacqua, 2015, p. 317) des pays porteurs de la candidature (Espagne, Grèce, Italie, Maroc) et du Centre international de hautes études agronomiques méditerranéennes (CIHEAM). Selon l'argumentaire développé par ces acteurs, le régime ainsi dénommé est un « héritage culturel immatériel » associant une « dimension gastronomique construite sur le goût, le plaisir et le partage ", à une " valeur de santé » et à des " qualités environnementales et paysagères » (Reguant-Aleix, 2012, p. 49).

Ce régime alimentaire a suscité le développement d'une terminologie associant les références des arts culinaires à l'univers socioculturel et géographique méditerranéen. Nous proposons d'analyser la circulation de l'information sur la diète méditerranéenne dans les modalités de mise en mots et de mise en discours dont elle fait l'objet dans la presse quotidienne nationale française, sur support imprimé ou numérique, dans la période de 2010 à 2019. En effet, la presse participe à la circulation de l'information et à la mise en visibilité des points de vue exprimés par certains acteurs dans l'espace public. Selon une conception communicationnelle de l'espace public (Pailliart, 1995), nous cherchons à évaluer les orientations contribuant à construire les représentations et les connaissances sur le régime méditerranéen en examinant les modes de médiatisation dont il fait l'objet dans ses logiques socioculturelles et professionnelles.

L'article est organisé en cinq sections. Nous présentons tout d'abord la méthodologie conçue pour appréhender le régime méditerranéen et la construction d'un corpus d'articles issus de la presse

2 La Convention pour la sauvegarde du patrimoine culturel immatériel a été adoptée lors de la 32e conférence générale de l'Unesco (Paris, 29/09-17/102003); elle est entrée en vigueur en octobre 2006. 
quotidienne nationale. Dans les sections suivantes, nous analysons son traitement médiatique selon des dimensions thématiques et lexicodiscursives, en association avec les univers socioculturels convoqués dans les articles.

\section{Construction du corpus et méthodologie}

La recherche des orientations identifiées dans les discours médiatiques de la presse nationale quotidienne française ${ }^{3} \mathrm{~s}$ ' appuie sur les traces constituées par les différents registres lexicaux qui y sont rattachés. Les contenus médiatiques de la presse nationale sont étudiés comme des cristallisations socio-discursives mettant au jour des perceptions, des représentations sociales et les imaginaires qu'elles convoquent au quotidien sur le régime méditerranéen.

La collecte des articles a été menée selon un cycle itératif sur les bases de données de médias Factiva et Europresse, ainsi que sur les sites des médias. L'équation de recherche " diète méditerranéenne ", élargie à des expressions proches « régime méditerranéen » et « régime crétois $^{4} »$, a été associée aux filtres concernant la langue (français), la zone géographique visée (France) et la période temporelle ( $\mathrm{du} 1^{\mathrm{er}}$ janvier 2000 au 31 mai 2019). La période retenue débute en 2000 pour observer la montée des préoccupations de différentes natures (cf. section 1). Une deuxième étape de curation du corpus a permis d'éliminer les articles traitant de manière incidente du régime méditerranéen. Le tableau 1 présente les principales caractéristiques du corpus collecté qui inclut les médias nationaux de la presse généraliste - Le Figaro (LF), Le Monde (LM), Libération (L), La Croix (LC) - et économique - Les Échos (LE), La Tribune (LT) - française bénéficiant de tirages élevés.

3 L'analyse du traitement médiatique du régime méditerranéen dans la presse régionale du sud de la France a fait l'objet d'une étude distincte : « Le régime méditerranéen dans la presse quotidienne locale : un modèle culturel alimentaire au cœur des problèmes sanitaires et environnementaux », Journées d'études « Crises sanitaires : expériences et politiques en santé humaine et animale », 27-29 mai 2019, Centre Norbert Elias, Marseille.

4 L'expression « régime crétois », fréquemment utilisée comme synonyme de « régime méditerranéen », fait référence aux travaux des nutritionnistes, notamment à l'étude du chercheur américain Ancel Keys (Keys, 1980) sur les bénéfices, pour la santé des habitants de l'île de Crète, d'une alimentation riche en huile d'olive, à dominante végétale et céréalière, (Dernini et al., 2012, pp. 73-84). 
Tableau 1. Liste des titres de presse nationale, tirage ${ }^{5}$ et nombre de signes des articles

\begin{tabular}{|l|l|l|l|l|}
\hline Corps d'articles & Tirage 2018 & $\begin{array}{l}\text { Nombre } \\
\text { d'articles }\end{array}$ & $\begin{array}{l}\text { Nombre } \\
\text { de mots }\end{array}$ & $\begin{array}{l}\text { Pourcentage } \\
\text { (en nombre } \\
\text { d'articles) }\end{array}$ \\
\hline Presse nationale française & & Total : 101 & & \\
\hline Le Figaro & 309492 & 36 & 23972 & $35,6 \%$ \\
\hline Le Monde & 302624 & 29 & 25177 & $28,7 \%$ \\
\hline Les Échos & 131413 & 17 & 9730 & $16,8 \%$ \\
\hline La Tribune & 66957 & 10 & 6859 & $9,9 \%$ \\
\hline Libération & 70501 & 5 & 2929 & $4,9 \%$ \\
\hline La Croix & 97009 & 4 & 2203 & $3,9 \%$ \\
\hline
\end{tabular}

La méthodologie repose sur l'apport conjoint de travaux sur l'étude des discours et la communication (Soulages, 2015). Le parcours interprétatif associe une approche quantitative et statistique (Née, 2017) à une approche qualitative, ancrées toutes deux dans l'étude des discours médiatiques, afin d'analyser, dans le corpus, les orientations de nature identitaire, gastronomique, culinaire, environnementales et sanitaires. Dans cette perspective, l'analyse thématique est associée à une analyse des univers lexicaux et de sens (section 3). L'appréhension d'ensemble et le repérage des caractéristiques du corpus orientent vers des questionnements et des investigations plus ciblées. Ces dernières ouvrent à la compréhension des phénomènes de médiation et de circulation de l'information d'actualité dans les médias appuyée sur les stratégies de nomination et les formes d'expression (Moirand, 2008) ainsi que sur les points de vue énonciatifs rapportés (Charaudeau, 2008).

\section{De la distribution thématique aux univers lexicaux et de sens}

Quelles sont les orientations thématiques concernant le régime méditerranéen les plus représentées dans les articles ? Ces orientations thématiques font référence à des thèmes ou à des points de vue récurrents dans les articles en association avec des acteurs engagés dans des rôles, des actions et des discours (Charaudeau, 2008, pp. 25-26).

5 ACPM, L'Alliance pour les Chiffres de la Presse et des Médias. En ligne : $\underline{\text { https:// }}$ www.acpm.fr/ 
L'analyse thématique a été réalisée par un mode de classement manuel sur la base de marqueurs linguistiques référentiels. En cas de présence de plusieurs thèmes, l'article a été catégorisé dans l'orientation thématique dominante. Dans cette catégorisation thématique, l'article publié constitue l'unité d'analyse et son niveau de granularité. Une analyse factorielle des correspondances, réalisée afin de vérifier la pertinence de ces thèmes, a confirmé que la distribution lexicale de ces catégories était bien distincte.

Cinq grandes orientations thématiques se dégagent de la lecture des articles. La figure 1 indique la répartition du nombre d'articles dans les catégories :

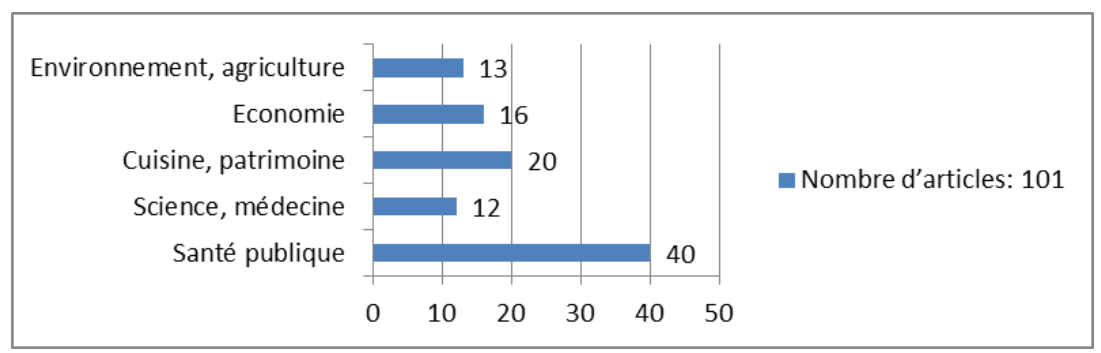

Figure 1. Orientations thématiques et distribution en $\%$ des articles

La figure 2 ci-après précise cette répartition par année. La distribution diachronique des catégories thématiques révèle les faits marquants en relation avec le régime méditerranéen ayant fait l'objet de l'attention médiatique. Les variations dans la présence dominante des catégories thématiques liées à la santé publique et à la médecine suivent et se font l'écho des rapports de santé publique nationaux et internationaux, ainsi que des publications des travaux de recherche. En 2010, 1'exclusivité de la catégorie concernant la cuisine et le patrimoine rend compte de l'inscription de la diète méditerranéenne sur la Liste représentative du patrimoine culturel immatériel de l'humanité de l'Unesco.

Sur la période temporelle retenue, les catégories thématiques dominantes s'avèrent centrées sur les problèmes de santé publique et les travaux scientifiques afférents. Les dimensions culinaire et patrimoniale ont également une présence affirmée ; les aspects économiques et environnementaux sont représentés mais avec une moindre occurrence. 


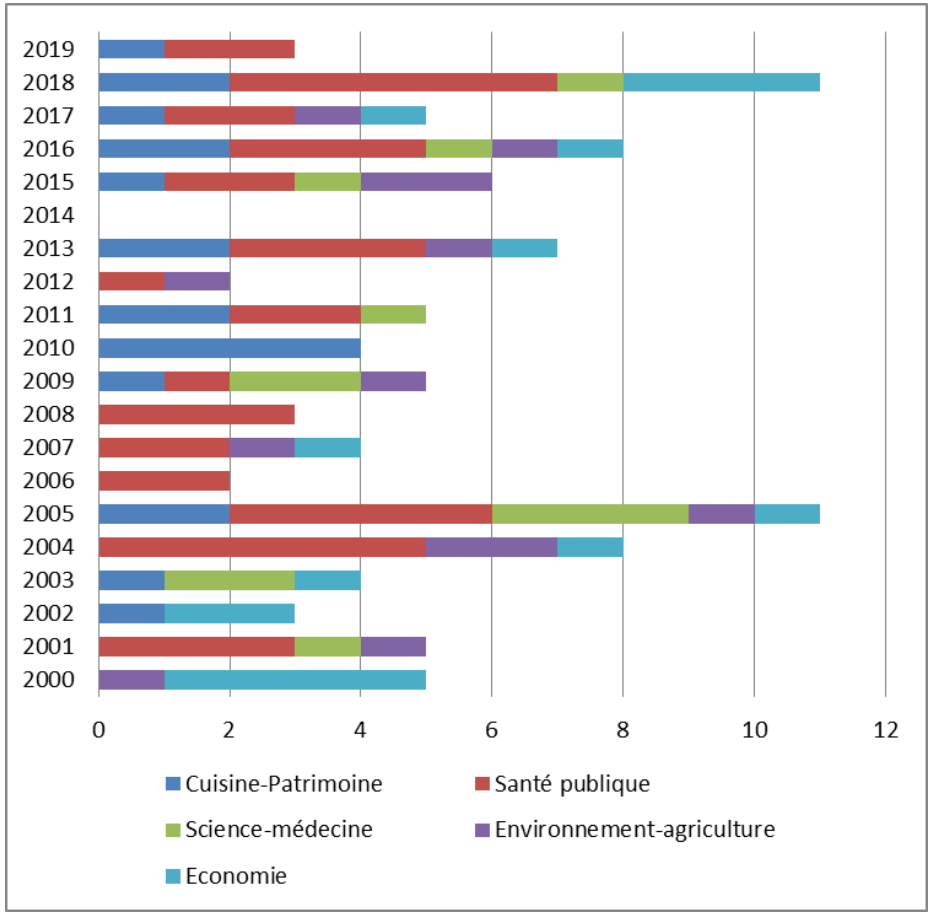

Figure 2. Répartition du nombre d'articles par année et par orientation thématique $^{6}$

Dans les discours médiatiques, comment s'articulent les catégories thématiques dominantes des articles identifiées ci-dessus et la diversité des univers lexicaux qui y sont rattachés ? Pour répondre à cette question, le repérage des catégories est complété par une analyse transversale sur l'ensemble du corpus. Cette analyse ouvre aux univers de sens qui y sont présents en s'appuyant sur les lexiques et les discours. La méthode statistique de classification descendante hiérarchique (CDH) conçue par Max Reinert (2008), permet d'identifier, à l'aide du logiciel Iramuteq ${ }^{7}$ (Ratinaud et al., 2009), les lexiques des discours étudiés. Elle repose sur le repérage, mené avec les méthodes et les outils d'analyse de données lexicométriques et textométriques, des formes

6 Selon les critères de collecte d'articles indiquées dans la section 1, il n'y a pas d'article retenu pour l'année 2014.

7 Iramuteq, logiciel sous licence GNU/GPL conçu par P. Ratinaud, laboratoire Lerass (EA 827), Université Toulouse 3. En ligne : http://www.iramuteq.org 
lexicales co-occurrentes à l'échelle de l'ensemble des articles. L'unité d'analyse est l'unité de contexte (UC) au sens de (Reinert, 2008), ce qui implique d'étudier le lexique de manière transversale aux articles de presse. L'identification des « mondes lexicaux » (Reinert, 2008) des articles associe, par le calcul statistique de co-occurrences, les dimensions de nature paradigmatique et syntagmatique de la séquence textuelle (Mayaffre, 2014, p. 29).

Des univers lexicaux émergent du traitement statistique des articles. Ils résultent du regroupement itératif des formes lexicales formant l'environnement linguistique immédiat (le co-texte) au sein des textes. Cinq classes ont ainsi été identifiées :

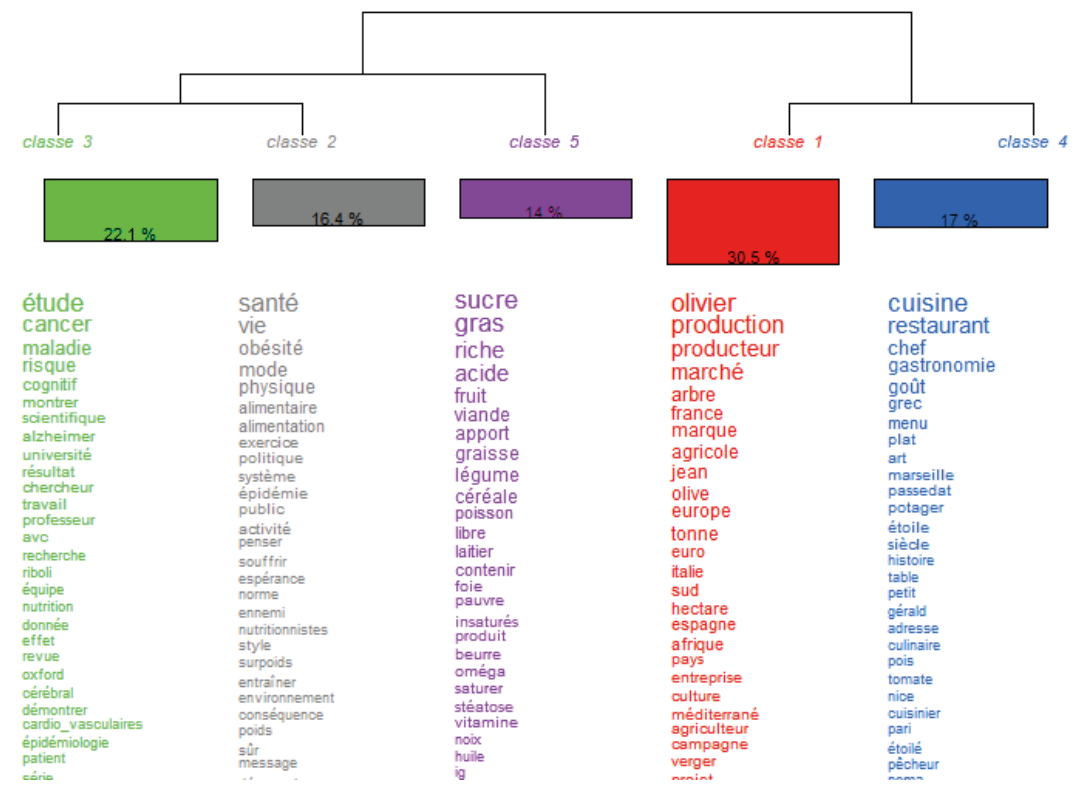

Figure 3. Dendogramme des classes (méthode de Reinert, logiciel Iramuteq)

Les univers lexicaux identifiés dans la figure 3 et répartis dans 5 classes sont calculés selon les critères de co-occurrence des termes dans le corpus. Ils complètent la classification d'orientation thématique réalisée précédemment en faisant apparaître des variations que nous allons analyser. La constitution des classes ouvre à une représentation cartographique de regroupements lexicaux présentée dans la figure 4 : 


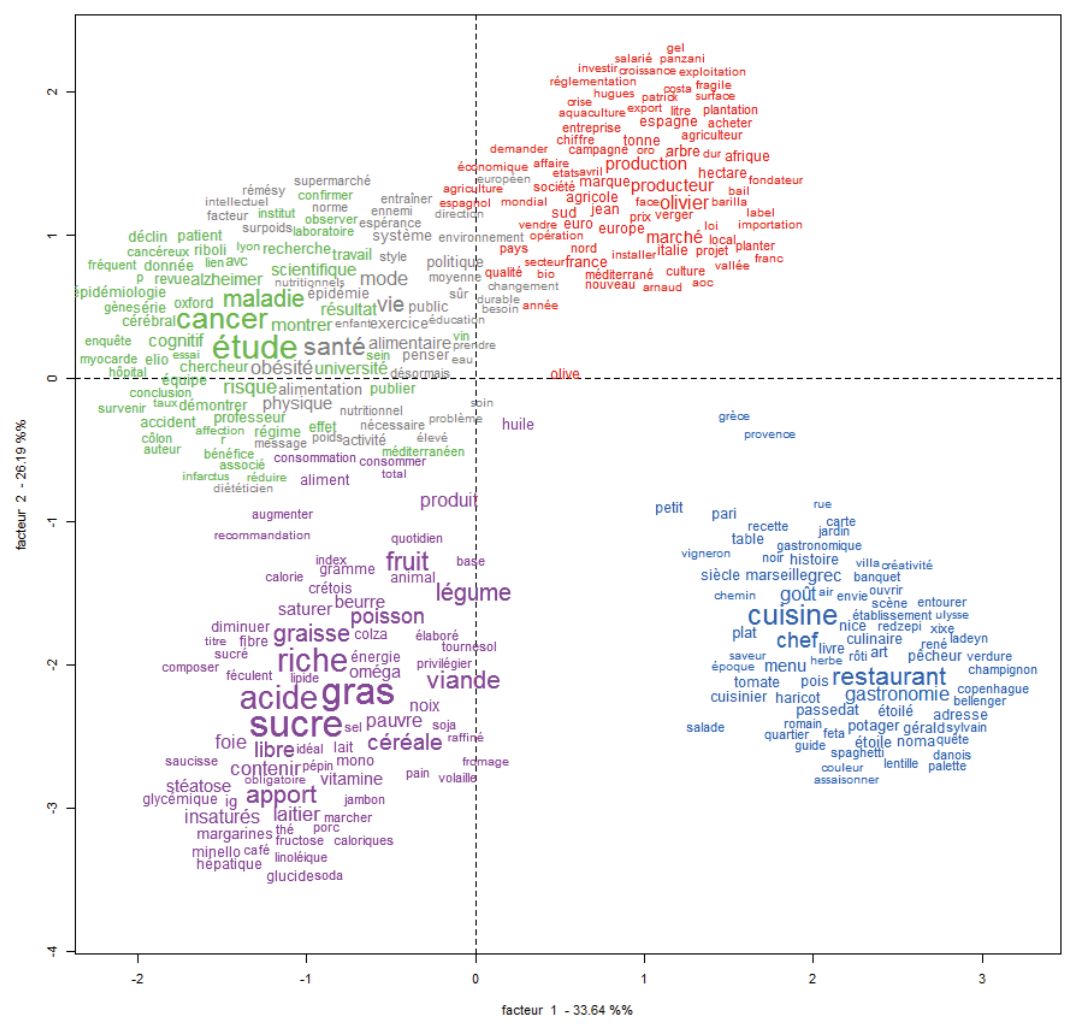

Figure 4. Analyse factorielle des correspondances (AFC) menée sur le tableau de contingence croisant lemmes et classes (méthode de Reinert, logiciel Iramuteq)

Les orientations thématiques et les univers lexicaux sont enrichis par un travail interprétatif mené au niveau des articles. Ce dernier permet d'appréhender les discours en relation avec les énonciateurs médiatisés faisant l'objet de discours directs ou rapportés dans leurs cadres communicationnels (Pincemin, 2012, p. 7). Le traitement médiatique du régime méditerranéen est ainsi mis en perspective avec les orientations patrimoniales et sanitaires qui ont participé de la construction socio-historique et à la mise en visibilité de ce mode alimentaire.

\section{Des traditions culinaires à la gastronomie végétale}

La représentation cartographique permet d'observer un groupement (en bleu) associant des dimensions à la fois culinaire, gastrono- 
mique et patrimoniale qui se détache nettement. Il rejoint les catégories thématiques sur la cuisine et le patrimoine (20 articles).

Dans les articles parus en 2010 (LF, LM, L), la reprise du communiqué de presse de l'Unesco décrit la diète méditerranéenne comme un « ensemble de compétences, de savoirs et de coutumes » concernant « les cultures, les récoltes, la pêche, l'élevage et surtout la façon de partager la table et de consommer les aliments ». Les «valeurs de l'hospitalité, du bon voisinage, du dialogue interculturel et de la créativité » formulées par l'Unesco sont reprises par les journalistes sans distance critique sur cette construction patrimoniale en regard de la grande diversité des pratiques alimentaires, sociales et culturelles rattachées aux pays concernés.

De plus, plusieurs travaux (CIHEAM, 2012) González Turmo et al., 2012) alertent sur les grandes disparités nord-sud, le stress hydrique en augmentation et l'impact environnemental négatif de modes de production agricoles hétérogènes, traditionnels et recourant fortement aux pesticides. Au-delà des discours sur la convivialité et le dialogue interculturel prônés à l'Unesco (Reguant-Aleix, 2012), ces travaux dénoncent également les fortes inégalités sociales des pays impliqués et les situations hétérogènes, parfois très inégalitaires, entre genres, origines sociales et nationales.

Le retour aux articles fait apparaître que ces aspects critiques sont absents des discours médiatiques sur la diète méditerranéenne. Ce régime alimentaire est présenté de manière valorisante en centrant l'attention sur des traditions culinaires par l'emploi d'un lexique mêlant histoire culturelle, aliments et géographie. Les traditions culinaires sont mises à l'honneur (LT, 26/07/2005) et la cuisine méditerranéenne est singularisée par des références à des savoir-faire nationaux associés à des aliments spécifiques. Ainsi, la cuisine italienne est louée pour sa capacité à « inventer un goût » et à rechercher des « saveurs naturelles », la diversité et la richesse des épices, des légumes, verdures et des pâtes. L'inscription des traditions culinaires dans l'histoire culturelle est utilisée comme un argument justifiant leur valorisation positive. La presse décrit des chefs qui voyagent « tel Ulysse » (LF, 19/05/2018), ou organisent un «banquet de Platon » (LF, 30/07/2011). Les articles citent les discours des chefs de la cuisine grecque qui vantent son « incroyable palette de saveurs, aussi variées que ses paysages » et son « histoire culinaire de quatre mille ans qui a sa source dans le bassin méditerranéen » (LF, 13/05/2018), à l'instar de la tapenade issue de la « recette de l'epityrum, à base d'olives vertes ou noires dénoyautées, assaisonnées 
d'huile, de vinaigre » dans l'Antiquité romaine (LT, 26/07/2005). Trois articles seulement portent un regard distancié sur cette construction patrimoniale. Ils soulignent ses enjeux de nature économique, identitaire et culturelle (LT, 26/07/2005) ainsi que les risques de dérives commerciales et politiques de $l^{\prime}$ ' instrumentation nationaliste » d'un " mythique noyau pur » des pratiques et coutumes nationales (L, 11/11/2010, 05/12/2013).

L'utilisation de termes issus de l'environnement marque l'entrée du régime méditerranéen dans les priorités écologiques actuelles en instituant le " règne de la gastronomie végétale » (LM, 16/12/2016). La notion de " naturalité » apparaît une fois dans l'ensemble du corpus médiatique dans un sens éloigné de son ancrage historique ${ }^{8}$. Elle provient d'une citation d'Alain Ducasse ayant « officialisé le concept de "naturalité" au Plaza Athénée, à Paris, depuis 2014, avec une carte consacrée aux légumes, céréales et poissons - où la viande ne figure plus » (LM, 16/12/2016). La carte d'Alain Ducasse est désormais dédiée aux " ingrédients modestes » érigés en " créations » (ibid.). Le chef Gérald Passedat (Le Petit Nice) déclare : « Je ne jure que par la Méditerranée ». Il décrit une recette " marine et végétale », emblématique de son restaurant gastronomique, qui « lie la nature et la Méditerranée et qui résonne avec le régime crétois plus qu'aucun autre plat chez moi » (LF, 18/03/2017). À la suite du chef renommé Alain Passard (Arpège), une nouvelle génération de chefs français et internationaux sont engagés dans une cuisine « végétale 》 (LM, 16/12/2016). Leurs propos sont repris sous forme de discours directs et de témoignages sur leurs liens profonds avec la nature. Le choix de la production agricole locale passe par des formes d'expression construites sur la création de mots ; ils reposent sur le procédé du figement et l'hybridation de lexiques issus de l'agriculture, la gastronomie et l'économie : les « produits ultralocaux » (ibid.), les « circuits ultracourts » ou le « concept de kilomètre zéro », les «primeurs en direct du potager » et les menus « en écho aux saisons » (ibid.). Ces « chefs fermiers » ont un jardin sur le toit de leur restaurant, un " restaurant-ferme », une ferme écologique ou urbaine (LM, 23/09/2015). L'un d'eux se déclare " chasseur-cueilleur ». Ils privilégient le terme « végétal » en glissant vers le " végétarisme » - en associant les produits carnés à la gastronomie française - tout en

$8 \mathrm{La}$ 《 naturalité », traduction du terme anglais " wilderness », fait référence à des écosystèmes qui ne présentent pas de traces d'influences d'activités humaines (Lecomte, 1999). 
affirmant des préoccupations écologiques et sanitaires. Ils insistent sur les nouvelles valeurs gustatives, « les saveurs », la « fraîcheur », la " légèreté » et la « croustillance » qu'ils attribuent aux légumes (LM, 16/12/2016).

Les préoccupations environnementales sont donc bien présentes dans les articles consacrés au patrimoine culinaire mais elles y apparaissent en relation avec la création et la gastronomie.

\section{La prédominance des préoccupations en santé publique}

Les catégories thématiques relevant de la santé publique (60 articles) et de la médecine (12 articles) sont les plus représentées. La représentation cartographique fait apparaître deux groupements formés par des lexiques de nature scientifique (en vert) et sanitaire (en gris) qui convergent vers des préoccupations de santé publique et forment un univers de sens distinct.

Cette prédominance médiatique peut trouver une explication dans la tradition de travaux scientifiques développés sur l'alimentation méditerranéenne. Ce modèle alimentaire a acquis une notoriété en raison de découvertes scientifiques (Nestle, 1995), les plus connues provenant du projet de recherche international « Seven Countries », mené à la fin des années 1950 par l'épidémiologiste américain Ancel Keys. La comparaison des régimes alimentaires de sept pays lui permit d'expliquer les différences entre les taux de mortalité provoquées par les accidents cardio-vasculaires. De nombreuses études scientifiques internationales confirmèrent la corrélation entre la combinaison de certaines catégories d'aliments et un régime calorique modéré avec l'augmentation significative de l'espérance de vie (Marques da Silva, 2015, p. 57). Depuis les années 1990, les travaux des nutritionnistes ont regroupé sous l'appellation « régime méditerranéen », les régimes dans lesquels l'olive est la principale source de graisse (Marques da Silva, 2015, p. 61). Au cours des années 2000, l'Organisation Mondiale de la Santé ${ }^{9}$ a alerté sur l'urgence d'une transition alimentaire visant à ralentir " l'épidémie » des maladies chroniques non transmissibles due en grande partie à un régime alimentaire déséquilibré.

9 Organisation Mondiale de la Santé (OMS). « La lutte contre les principales maladies en Europe : problèmes et solutions », Copenhague, 11/09/2006. " Lutte contre les maladies non transmissibles : mise en œuvre de la stratégie mondiale », document A61/8, Rapport du Secrétariat. 18/04/2008. 
Le régime alimentaire méditerranéen, lié à la santé publique dans la lutte contre les maladies chroniques, est devenu une question de politique sanitaire relayée par les médias nationaux. Ce régime est décrit comme permettant notamment de réduire les risques d'accidents cardio-vasculaires, de cancer, de dépression, d'obésité ou de dégénérescence cérébrale. Selon un système énonciatif construit sur une prise en charge énonciative (Rabatel, 2012) forte, les articles sont émaillés des témoignages et des prises de position des nombreux professeurs et chercheurs rattachés à des universités et à des laboratoires dans les domaines de la nutrition, de la cancérologie, de la biochimie, de la neurologie, de la gériatrie, de la psychiatrie : « Des chercheurs américains ont mis en évidence les propriétés de l'huile d'olive pour lutter contre le cancer du sein. [...] "Les résultats de nos recherches tendent aussi à confirmer les études épidémiologiques ayant montré que le régime alimentaire dit méditerranéen, riche notamment en huile d'olive, a des effets protecteurs contre le cancer, les maladies cardio-vasculaires et le vieillissement", a noté le docteur Menendez » (LM, 10/01/2005). « Des chercheurs de l'Inserm et de l'université de Montpellier viennent de le confirmer dans une étude publiée par la revue Molecular Psychiatry [...]. Les conclusions des chercheurs sont que l'adoption du régime méditerranéen - alimentation riche en fruits et en légumes, poisson et céréales - diminue de $33 \%$ le risque de dépression » (LE, 15/10/2018). Ce sont les acteurs de la recherche et les praticiens au quotidien qui sont mis en valeur dans les articles. Leurs propos sont repris en utilisant la forme des discours directs ou indirects tout en précisant leurs titres universitaires afin de renforcer la légitimité d'une conception nutritionnelle du régime méditerranéen : " "Les résultats de notre étude montrent l'importance de nos habitudes alimentaires dans la survenue de troubles dépressifs et encouragent à généraliser le conseil nutritionnel lors des consultations médicales", conclut Tasnime Akbaraly, chercheuse à l'Inserm chargée de l'étude » (ibid.). Lors de la conférence internationale sur le rôle des fruits et légumes dans la prévention de l'obésité (17-19/2007, Bruxelles), Le professeur Elio Riboli, spécialiste de nutrition et du cancer, responsable de la division épidémiologie à l'Imperial College de Londres, a rappelé : « les fruits et légumes sont essentiels pour la santé ». " La première étude épidémiologique sérieuse sur l'effet protecteur des fruits et légumes est américaine et remonte aux années 1930. Puis, après la Seconde Guerre mondiale, la comparaison de la mortalité par infarctus du myocarde dans différents pays a fait émerger l'idée que le régime méditerranéen (fruits et légumes et huile 
d'olive) était bon pour protéger des maladies cardio-vasculaires » (LM, 21/04/2007).

La description des aliments qui composent le régime méditerranéen dans ses caractéristiques nutritionnelles apparaît dans la classe 5 (en violet) du traitement statistique. Cette classe rend compte du lexique utilisé par les professionnels de santé publique qui détaillent les apports caloriques, les molécules, les vitamines et les fibres des divers aliments recommandés (céréales, fruits, légumes, poissons...) dans un modèle alimentaire équilibré : « [...] que mangent donc les Crétois pour avoir un cœur si résistant? Principalement des céréales, des légumes secs (pois chiches, fèves) et verts, très peu de viande, un peu de poisson, un peu de fromage de brebis et de l'huile d'olive (riche en acide oléique) » (LF, 27/12/2001). Une diététicienne nutritionniste dans le service d'hépato-gastro-entérologie d'un CHU explique que, dans les pratiques alimentaires, « corriger de mauvaises habitudes peut sauver un foie »; elle conseille « les matières grasses riches en acides gras mono-insaturés et polyinsaturés, surtout les oméga 3 comme l'huile d'olive, de lin, de colza, les oléagineux, les poissons en général et plus précisément la sardine, le maquereau, le hareng, le saumon. On en revient finalement au régime méditerranéen » (LF, 28/05/2018).

En revanche, les journalistes ne donnent pas la parole aux individus qui sont présentés dans un rôle passif : ce sont les "patients » et « malades » cités dans les cohortes des recherches scientifiques. Ce sont également les nombreuses victimes des maladies (cancers, diabètes, problèmes cardiaques, obésité...) en partie favorisées par un régime alimentaire déséquilibré éloigné du modèle méditerranéen. Les « consommateurs » sont décrits dans les évolutions de leurs comportements alimentaires et leurs réactions face à des « messages nutritionnels contradictoires » émanant des lobbies et contre-lobbies (LF, 28/05/2018), dans leurs demandes de sécurité alimentaire (LM, 04/03/2000). L'information auprès des adultes et des jeunes, qui préconise l'exercice physique et un modèle nutritionnel favorisant les légumes et de fruits bénéfiques à leur santé est une priorité affichée de la politique de santé européenne (LF, 26/05/2005). Enfin, le rôle de « citoyens » leur octroie de manière floue une capacité de décision et d'action concernant leur propre santé (LM, 28/04/2015, 27/02/2013) sans discuter des moyens d'information dont ils disposent. 


\section{Des préoccupations agricoles et économiques}

Dans la représentation cartographique, le groupement de la classe 1 (en rouge) fait ressortir les aspects agricoles et économiques rattachés au régime méditerranéen. La catégorie thématique sur l'environnement (12 articles) fait aussi apparaître cette approche qui est présente, de manière minoritaire et sans augmentation notable au cours des dernières années dans le corpus, comme le montre la figure 2. Une place particulière est réservée à l'huile d'olive qui apparaît de manière transversale, comme l'indique sa position sur la carte, dans 36 articles du corpus. « L'huile, crème d'une alimentation saine », consacre ainsi « la vogue de la diète méditerranéenne » : « du poisson, du pain, des légumes, des fruits, un verre de vin éventuellement et un soupçon d'huile d'olive, omniprésente sur le pourtour méditerranéen » (LF, 17/04/2006).

L'affirmation d'un style d'alimentation « méditerranéen » pour des besoins de patrimonialisation fait apparaître, dans ses aspects ethnoanthropologiques, de "nombreuses et diverses cultures alimentaires méditerranéennes » (Reguant-Aleix, 2012, pp. 38-40), ce qui permet d'appréhender les difficultés à les constituer en « patrimoine » commun. «L'identité alimentaire » des pays méditerranéens est analysée dans les travaux des anthropologues en étant « perçue plutôt en termes de céréales et de légumes » avec trois ingrédients de base (le blé, l'olivier, la vigne), auxquels s'ajoutent les légumes secs et verts, les fruits, le sucre et le miel, le lait et le fromage, et en moindre quantité la viande et le poisson. Au-delà d'un modèle nutritionnel, ce régime est une « réalité dans laquelle interviennent la production, la commercialisation, la consommation, la convivialité, les rituels et la symbolique alimentaire de la région méditerranéenne, ainsi que ses cuisines et les denrées ellesmêmes » (González Turmo, 2012, p. 122).

Ces différents aspects apparaissent dans les articles en y ajoutant la promotion d'un modèle de culture durable et locale présenté comme porteur de pratiques innovantes. En premier lieu, la production agricole méditerranéenne est abordée en relation avec la gastronomie. Les chefs travaillent « en direct » avec des producteurs valorisés pour leur engagement dans des modèles de culture durable. Les formes d'expression portent cette évolution par des termes techniques rattachés à l'agriculture biologique et par la création de mots rapprochant les métiers et les produits hérités du passé de nouvelles pratiques. Ces producteurs sont décrits comme des "néocultivateurs » et des "néoviticulteurs " (le préfixe " néo » soulignant la reprise de métiers anciens sous une 
forme renouvelée) qui sont « de plus en plus nombreux à travailler en permaculture et en biodynamie », des « producteurs et des vignerons d'excellence » (LF, 23/03/2019). Leurs produits (feta, huile d'olive, vins, miels...) sont associés à leurs lieux de production. Des « agriculteurs, cueilleurs, producteurs maraîchers en bio »sont décrits comme « ultracréatifs »(LM, 16/12/2016). Selon un procédé métonymique, les qualités des productions deviennent celles des producteurs décrits comme des « pêcheurs durables » « qui redonnent le goût des saisons à des variétés de poissons oubliées » (LF, 23/03/2019) ou une « nouvelle race de producteur d'huile d'olive » spécialisé (LF, 22/08/2003).

En second lieu, les producteurs agricoles de produits méditerranéens sont médiatisés comme les acteurs de la scène économique abordée à l'échelle des pays et des parts de marché européens. Le secteur oléicole est en particulier essentiel à l'agriculture des pays d'Europe $\mathrm{du}$ sud (Espagne, Italie, Grèce) qui sont des producteurs mondiaux d'huile d'olive (LM, 12/04/2013). Des producteurs locaux sont mis en exergue, tels les «producteurs d'olives de la vallée des Baux, au pied des Alpilles » et leur spécialité locale d'olives cassées (LT, 25/10/2000) ou la qualité française des huiles « vierge » ou « vierge extra »-obtenue par pression du fruit et sans ajout d'élément chimique (L, 23/05/2000). Les stratégies des entreprises européennes, voire internationales, sur un marché très concurrentiel sont aussi analysées (LT, 25/10/2000). Enfin, le régime méditerranéen est aussi présenté au service du développement socio-économique des territoires, à l'instar de l'événement gastronomique et culturel, "Marseille Provence, année de la Gastronomie ", organisé en 2019 par la ville de Marseille et la Région Sud avec le parrainage du chef Gérald Passedat (LF, 23/03/2019).

\section{Conclusion}

Au terme de cette analyse conjointement quantitative et qualitative appuyée sur des catégories thématiques et des univers lexico-discursifs, plusieurs tendances se dégagent concernant la distribution et la circulation de l'information d'actualité sur le régime méditerranéen dans la presse nationale française. Sur une période de vingt ans, il apparaît comme un thème d'intérêt suivi dans la durée selon cinq orientations thématiques dominantes en bénéficiant de la diffusion de ces titres.

Au sein des univers socioprofessionnels médiatisés, les problèmes de santé publique en relation avec les recherches liés à l'alimentation prédominent. Dans ce domaine, un discours médical de santé publique 
appuyé sur des travaux scientifiques promeut un modèle fondé sur la normalisation des comportements alimentaires, la nutrition et la prévention. La médiatisation d'une culture culinaire méditerranéenne reprend les discours d'inscription de la « diète méditerranéenne » au patrimoine culturel immatériel de l'humanité de l'Unesco concernant la nature et les cultures alimentaires construites sur la sociabilité, les saveurs et les savoir-faire culinaires des pays de la Méditerranée. Cette construction patrimoniale est peu remise en cause dans les discours médiatiques en dépit de l'hétérogénéité des cultures alimentaires, des fortes inégalités socio-économiques et des préoccupations environnementales croissantes. Les traditions culinaires sont aussi médiatisées comme un champ d'inspiration pour une " gastronomie végétale » reposant sur la création et l'innovation de chefs renommés. Un univers lexical et de sens autour des végétaux émerge, porteur de représentations et de valeurs positives à l'intersection de la gastronomie, associée à un plaisir gustatif renouvelé, et de préoccupations écologiques. La promotion de choix favorisant des modèles de production agricole durable et locale côtoient les stratégies industrielles et économiques des producteurs agricoles sur un marché européen concurrentiel.

\section{Références}

Bevilacqua, S. (2015). La fortune de la diète méditerranéenne. De la médicalisation à la patrimonialisation, Palaver 4(1), 315-348. doi : https://doi.org/10.1285/ i22804250v4i1p315

Charaudeau, P. (dir.) (2008). La Médiatisation de la science. Bruxelles : De Boeck.

Dernini, S., Berry, E., Bach-Faig, A., Belahsen, R., Donini, L., Lairon, D., Lluís Serra-Majem, L., Cannella, C. (2012). Un modèle alimentaire construit par les scientifiques. Dans CIHEAM (dir.), MeditTERRA 2012, La diète méditerranéenne pour un développement régional durable (pp. 73-84). Paris : Presses de Sciences Po.

González Turmo, I. (2012). La diète méditerranéenne : consommation, cuisines et pratiques. Dans CIHEAM (dir.), MediTERRA 2012, La diète méditerranéenne pour un développement régional durable (pp. 121-136). Paris : Presses de Sciences Po.

González Turmo \& I., Medina, F.-X. (2012). Défis et responsabilités suite à la déclaration de la diète méditerranéenne comme patrimoine culturel immatériel de l'humanité (Unesco), Revue d'ethnoécologie 2. doi : https://doi.org/10.4000/ethnoecologie. 957

Keys, A. (1980). Seven Countries. A Multivariate Analysis of Death and Coronary Disease. Cambridge : Harvard University Press.

Lecomte, J. (1999). Réflexions sur la naturalité, Le Courrier de l'environnement de l'INRA, Paris : Institut national de la recherche agronomique Délégation permanente à l'environnement 37, août 1999, 5-10. Disponible à : https://hal.archives-ouvertes. fr/hal-01203602/file/C37Lecomte.pdf 
Marques da Silva, A. J. (2015). La Diète méditerranéenne. Discours et pratiques alimentaires en Méditerranée. Vol. 2, Coll. Questions alimentaires et gastronomiques, Paris : L'Harmattan.

Mayaffre, D. (2014). Plaidoyer en faveur de l'analyse de données co(n)textuelles. Parcours cooccurrentiels dans le discours présidentiel français (1958-2014). Dans JADT. $12^{e}$ Journées internationales d'Analyse statistique des Données Textuelles, Paris. Disponible à : http://lexicometrica.univ-paris3.fr/jadt/jadt2014/01ACTES/01-JADT2014.pdf

Moirand, S. (2008). Les Discours de la presse quotidienne. Observer, analyser, comprendre. Coll. Linguistique nouvelle, Paris : PUF.

Née, E. (dir.) (2017). Méthodes et outils informatiques pour l'analyse des discours, Rennes : Presses universitaires de Rennes.

Nestle, M. (1995), Mediterranean Diets, Historical and Research Overview, American Journal of Clinical Nutrition 61, 1313-1320. doi : https://doi.org/10.1093/ ajcn/61.6.1313S

Pailliart, I. (1995). L'Espace public et l'emprise de la communication. Grenoble : Ellug.

Pincemin, B. (2012). Sémantique interprétative et textométrie, Texto! Textes et cultures 17(3), 1-21. Disponible à : http://www.revue-texto.net/docannexe/file/3049/pincemin_texto11.pdf

Poulain, J.-P. (2002). Manger aujourd'hui. Attitudes, normes et pratiques. Toulouse : Privat.

Rabatel, A. (2012). Les relations Locuteur/Énonciateur au prisme de la notion de voix. Dans Gournay, L. \& Dufaye, L., Les Théories de l'énonciation : Benveniste après un demi-siècle. Arts et Savoirs 2. doi : https://doi.org/10.4000/aes.510

Ratinaud, P. \& Déjean, S. (2009). Iramuteq. Implémentation de la méthode Alceste d'analyse de texte dans un logiciel libre. Dans Modélisation appliquée aux sciences humaines et sociales, Toulouse, Université Toulouse 2 Le Mirail.

Reguant-Aleix, J. (2012). « Chapitre 1. La diète méditerranéenne: donner un nom au futur ». Dans CIHEAM (dir.), MediTERRA 2012, La diète méditerranéenne pour un développement régional durable (pp. 27-51). Paris, Presses de Sciences Po (P.F.N.S.P.).

Reinert, M. (2008). Mondes lexicaux stabilisés et analyse statistique de discours. Dans JADT. 9e Journées internationales d'Analyse statistique des Données Textuelles, Lyon. Disponible à : http://lexicometrica.univ-paris3.fr/jadt/jadt2008/pdf/reinert. pdf

Soulages, J.-C. (dir.) (2015). L'Analyse du discours : sa place dans les sciences $d u$ langage et de la communication. Hommage à Patrick Charaudeau. Coll. Rivages linguistiques, Lyon : Presses universitaires de Lyon. 


\section{(c) (1) (-) $\Theta_{\mathrm{BY}}$ NC ND Publié sous la licence Creative Common}

«Attibution - pas d'utilisation Commerciale - Pas de Modification 4.0. International» (CC BY-NC-ND) 